\title{
Causes of Morbidity and Mortality of Wildlife Species Presented to a Wildlife Clinic in East Tennessee, USA, 2000-2011
}

\section{Schenk $A^{*}$}

\author{
Lauderdale Veterinary Specialists, USA
}

*Corresponding author: Schenk A, Lauderdale Veterinary Specialists, USA, E-mail: schenktn@gmail.com

Citation: Schenk A (2017) Causes of Morbidity and Mortality of Wildlife Species Presented to a Wildlife Clinic in East Tennessee, USA, 2000-2011. J Vet Sci Ani Husb 5(4): 404. doi: 10.15744/2348-9790.5.404

Received Date: November 16, 2017 Accepted Date: December 27, 2017 Published Date: December 29, 2017

\begin{abstract}
To determine the causes of wildlife morbidity and mortality in East Tennessee, we performed a retrospective analysis using 14,303 records from cases presented to the wildlife clinic of the University of Tennessee between 2000 and 2011. The cases were first categorized into herptile (includes reptiles and amphibians), mammal, or avian and then classified into 1 of 20 groups based on the primary admitting/presenting sign. The top reasons for presentation were trauma, found abandoned/orphaned, hit by automobile, cat-related injury, and dog-related injury. Overall mortality was $45.3 \%$ for the herptile cases, $57.6 \%$ for mammal cases, and $65.1 \%$ for avian cases. There are a variety of reasons animals were presented to the clinic, and some appear to be attributed to anthropogenic factors such as automobiles $(n=1253)$ and habitat destruction $(n=218)$, while the origin of others, such as trauma and disease remain unknown. This study confirms the importance of monitoring wildlife morbidity and mortality as an indicator of ecosystem health and of focusing efforts to reduce the anthropogenic threat on native habitats and resident wildlife populations.
\end{abstract}

Keywords: Wildlife; Morbidity; Anthropogenic; Biological Corridors

\section{Introduction}

Around the world, wildlife species and the ecosystems they inhabit are evolving and shifting in an attempt to adapt to human influences and environmental change. The factors dictating these changes are diverse and numerous, making it difficult to separate the effect each has on species survivorship. Increased morbidity and mortality of wildlife can be attributed to a variety of factors, including infectious diseases and environmental toxins, as well as exposure to such human activities as land development, predation by domestic pets, and automobile traffic [1-5].

As the urbanization of areas with native wildlife continues to increase, numerous pressures are exerted on the natural structures of those habitats. For example, infringing development may not only increase exposure between wildlife species, domestic animals, and invasive species, but may also increase wildlife's exposure to novel pathogens, leading to what Daszak et al. call "pathogen pollution" (2000). Furthermore, fragmentation of natural landscapes and, thus, wildlife populations poses additional dangers. Dividing wildlife species into smaller subpopulations threatens the conservation of biodiversity by limiting the genetic variation within breeding groups and decreasing breeding ground area [6,7]. Native habitats are becoming more divided and isolated by land development $[3,7,8]$, and it is possible that this fragmentation may lead to an increase in wildlife morbidity and mortality due to new infectious diseases. Although some of this increased morbidity and mortality can be directly attributed to habitat disruption, it may also be indirectly linked to anthropogenic-induced climate change resulting in the shift of geographic ranges of certain parasites and predators $[9,10]$. Non-native species carry with them non-native pathogens as well as the ability to alter the balance of predator/prey populations in established ecosystems. This introduction of new disease and new predators can have deleterious effects on wildlife species.

Clearing and isolating wildlife habitats may also lead to high-traffic roadways, which increase roadside automobile-related mortalities, noise, environmental pollution, and disturbance stress on the surrounding wildlife [11]. The construction of other infrastructure can change the flow of water runoff thereby increasing flooding and further deteriorating the habitable environment. The establishment of neighborhoods and housing communities will likely also increase the number of domestic animals in the area, which provides an unnatural predator stress on nearby native species.

Regardless of whether these increasing threats to wildlife originate directly or indirectly from anthropogenic effects, or as a result of disease spread, it is important to understand the extent of their impact. One study found that trauma and infection were the 
main reasons eastern box turtles were presented to a wildlife clinic, and this study seeks to reveal whether these causes dominate on a larger, more comprehensive scale [12]. Similar studies were conducted to investigate reasons wild raptors and reptiles were admitted to wildlife rehabilitation centers, and both studies found evidence of anthropogenic origins of trauma [13,14]. The cases of trauma resulting from direct or indirect human impact, however, were not further subcategorized. The purpose of this study was to investigate the reasons wildlife were presented to a veterinary medical center to determine the greatest causes of morbidity and mortality.

\section{Methods}

We reviewed 14,943 records from wildlife cases that presented to the University of Tennessee between January 2000 and November 2011. This number includes cases in which "Dead on Arrival" or "Euthanasia" were the only details given for the reason for presentation. It also includes rechecks and cases in which there was insufficient data for categorization. Because of the lack of sufficient data in these cases, they were removed from the dataset. The 14,303 records remaining included species or species group ("songbird" being the most detailed animal group indicated on many avian records), and these species or species groups were classified according to type of animal (herptile, mammal, or avian). The cases were then further grouped into 20 categories according to their primary admitting/presenting sign (A/PS), as shown in Table 1. These signs were derived from information provided by the person admitting the animal and do not represent final diagnoses made by the clinician, but they do provide detailed information about the patient's condition.

\begin{tabular}{|c|c|c|c|}
\hline $\begin{array}{c}\text { ADMITTING/PRESENTING } \\
\text { COMPLAINT }\end{array}$ & HERPTILE & MAMMAL & AVIAN $^{*}$ \\
\hline (Unknown) & $10(0.00)^{*}$ & $66(0.41)^{*}$ & $111(0.55)^{*}$ \\
\hline Agonal/Respiratory & $6(0.33)$ & $20(0.85)$ & $25(0.56)$ \\
\hline Anemia/Dehydration & $1(0.00)$ & $23(0.26)$ & $7(0.71)$ \\
\hline Behaviorally Abnormal & $10(0.30)$ & $89(0.67)$ & $68(0.60)$ \\
\hline Cat Related Injury & $12(0.33)$ & $1123(0.65)$ & $809(0.71)$ \\
\hline Congenital/Growth & $2(0.00)$ & $0(\mathrm{~N} / \mathrm{A})$ & $3(1.00)$ \\
\hline Disease Suspected & $9(0.11)$ & $39(0.77)$ & $19(0.68)$ \\
\hline Dog Related Injury & $51(0.39)$ & $597(0.58)$ & $245(0.70)$ \\
\hline Foreign Body/Substance & $1(0.00)$ & $6(0.50)$ & $5(0.40)$ \\
\hline Ingestion & $39(0.23)$ & $1442(0.30)$ & $1240(0.47)$ \\
\hline Found/Abandoned/Orphaned & $14(0.07)$ & $6(0.83)$ & $8(0.63)$ \\
\hline Growth/Mass/Cyst & $0(\mathrm{~N} / \mathrm{A})$ & $133(0.27)$ & $85(0.41)$ \\
\hline Habitat/Nest Destruction & $275(0.59)$ & $495(0.84)$ & $483(0.66)$ \\
\hline Hit by Car & $0(\mathrm{~N} / \mathrm{A})$ & $2(0.50)$ & $7(0.57)$ \\
\hline Malnourished & $0(\mathrm{~N} / \mathrm{A})$ & $71(0.85)$ & $37(0.81)$ \\
\hline Neurologic & $0(\mathrm{~N} / \mathrm{A})$ & $24(0.42)$ & $16(0.63)$ \\
\hline Parasites & $3(0.00)$ & $0(\mathrm{~N} / \mathrm{A})$ & $0(\mathrm{~N} / \mathrm{A})$ \\
\hline Translocation & $11(0.18)$ & $100(0.77)$ & $56(0.71)$ \\
\hline Trauma & $382(0.44)$ & $1517(0.70)$ & $4492(0.69)$ \\
\hline Reproduction Complication & 826 & 5761 & 7716 \\
\hline Sickly/Ill & $8(0.25)$ & $0(\mathrm{~N} / \mathrm{A})$ \\
\hline
\end{tabular}

"Most common herptiles include chelonia, snakes, amphibians, lizards; most common mammals include rabbits, squirrels, opossums, racoons; most common avian include songbirds, coulumbiformes, raptors, waterfowl *Case fatality rate is in parentheses

Table 1: Frequency of common admitting/presenting signs for herptile, mammal, and avian species presented to a wildlife clinic in East Tennessee between 2000-2011

If cases contained more than one primary A/PS, they were categorized according to the most detailed sign in the description. In cases where multiple signs of equal detail were provided, the case was categorized according to the sign first mentioned. Most of these A/PSs are self-evident; others were grouped as follows: If case notes indicated that there was impaired motor function, a wound or wound abscess, air sac rupture (in the case of 1 songbird), mention of the animal being found entrapped in barbed wire, trap etc., or cases of drowning were all categorized as trauma. Cat related, dog related, and automobile related cases were separated out from trauma in order to show their frequency. Any time "hit by car" was indicated, the animal was placed in the hit by automobile category. If the animal was found "in" the road, it was categorized as hit by automobile, but those that were found "near road" were placed under found/abandoned/orphaned. If a non-specific "dog/cat" or "cat/dog" attack was noted, the patient was categorized by the animal mentioned first. Behaviorally abnormal cases included those that mentioned lethargy, anorexia, aggressive behavior, 
unresponsiveness, weakness, or general statements of acting abnormally. Habitat destruction included nest destruction and any mention of trees being cut down, but cases in which nests fell or were blown from a tree and cases in which a tree fell (but not from being cut down) were categorized as simple found/abandoned/orphaned cases. The category "Disease suspected" includes cases of bloating, scale/feather sloughing, diarrhea, vomiting, infection, fever, as well as any mention of suspected disease, whereas cases without specifics about illness were categorized as sickly/ill. A general description of discharge, missing feathers, and inability to urinate or defecate were all categorized as unknown.

The number of cases in each category was evaluated, and the top four A/PSs were recorded for herptile and avian groups, and the top five A/PSs were recorded for mammals. A drop-off in case frequency after these top categories occurred and there was a high frequency of automobile related cases appearing in all three animal groups. Outcomes (alive, dead on arrival [DOA], died, or euthanized) were recorded for these top A/PSs, mortality rates were calculated for the top four species affected in the three groups, and case fatality rates were calculated for each A/PS. Finally, cases were sorted by year of presentation in order to determine the presence of any temporal pattern.

\section{Results}

For the $20 \mathrm{~A} / \mathrm{PSs}$, the case frequencies ranged from 0 to 4,492 for each animal group, and the case fatality rates ranged from 0.0 to 1.0 (Table 1). The top four or five A/PSs for the herptile, mammal, and avian groups were determined, and the percentages of cases for each are shown in Table 2. The species most commonly presented and their outcomes are shown in Table 3. Specifically, chelonians with trauma were the most numerous of the herptile cases resulting in death (169 cases), however an additional 163 herptile cases specified death due to being hit by an automobile. Rabbits with cat-related trauma were the most numerous of the mammal cases resulting in death (537), and songbirds with trauma of unknown origin were the most numerous of the avian cases resulting in death (2332).

\begin{tabular}{|l|c|c|c|c|c|c|c|c|c|c|c|}
\hline & \multicolumn{2}{|c|}{$\mathrm{n}(\%)^{* *}$} & \multicolumn{2}{c|}{ ALIVE (\%) } & \multicolumn{2}{c|}{ DOA (\%) } & \multicolumn{2}{c|}{ DIED (\%) } & \multicolumn{2}{c|}{ EUTHAN (\%) } & CFR \\
\hline \multicolumn{1}{|c|}{ HERP $^{*}$} & 826 & $(100.0)$ & 452 & $(54.7)$ & 6 & $(0.7)$ & 56 & $(6.8)$ & 312 & $(37.8)$ & \\
\hline 1. Trauma* & 392 & $(47.5)$ & 213 & $(54.3)$ & 3 & $(0.8)$ & 21 & $(5.4)$ & 145 & $(37.0)$ & 0.431 \\
\hline 2. Hit by Car & 275 & $(33.3)$ & 112 & $(40.7)$ & 3 & $(1.1)$ & 25 & $(9.1)$ & 135 & $(49.1)$ & 0.593 \\
\hline 3. Dog Related & 51 & $(6.2)$ & 31 & $(60.8)$ & 0 & $(0.0)$ & 3 & $(5.9)$ & 17 & $(33.3)$ & 0.392 \\
\hline $\begin{array}{l}\text { 4. Found/Abandoned/ } \\
\text { Orphaned }\end{array}$ & 39 & $(4.7)$ & 30 & $(76.9)$ & 0 & $(0.0)$ & 2 & $(5.1)$ & 7 & $(17.9)$ & 0.231 \\
\hline \multicolumn{1}{|c|}{ MAMMAL } & 5761 & $(100.0)$ & 2445 & $(42.4)$ & 65 & $(1.1)$ & 504 & $(8.7)$ & 2747 & $(47.7)$ & \\
\hline 1. Trauma* & 1517 & $(26.3)$ & 458 & $(30.2)$ & 16 & $(1.1)$ & 118 & $(7.8)$ & 925 & $(61.0)$ & 0.698 \\
\hline $\begin{array}{l}\text { 2. Found/Abandoned/ } \\
\text { Orphaned }\end{array}$ & 1442 & $(25.0)$ & 1009 & $(70.0)$ & 6 & $(0.4)$ & 87 & $(6.0)$ & 340 & $(23.6)$ & 0.300 \\
\hline 3. Cat Related & 1123 & $(19.5)$ & 391 & $(34.8)$ & 9 & $(0.8)$ & 167 & $(14.9)$ & 556 & $(49.5)$ & 0.652 \\
\hline 4. Dog Related & 597 & $(10.4)$ & 253 & $(42.4)$ & 13 & $(2.2)$ & 52 & $(8.7)$ & 279 & $(46.7)$ & 0.576 \\
\hline 5. Hit by Car & 495 & $(8.6)$ & 81 & $(16.4)$ & 12 & $(2.4)$ & 32 & $(6.5)$ & 370 & $(74.7)$ & 0.836 \\
\hline \multicolumn{1}{|c|}{ AVIAN } & 7716 & $(100.0)$ & 2695 & $(34.9)$ & 88 & $(1.1)$ & 1251 & $(16.2)$ & 3682 & $(47.7)$ & \\
\hline 1. Trauma* & 4492 & $(58.2)$ & 1390 & $(30.9)$ & 52 & $(1.2)$ & 733 & $(16.3)$ & 2317 & $(51.6)$ & 0.691 \\
\hline $\begin{array}{l}\text { 2. Found/Abandoned/ } \\
\text { Orphaned }\end{array}$ & 1240 & $(16.1)$ & 652 & $(52.6)$ & 9 & $(0.7)$ & 157 & $(12.7)$ & 422 & $(34.0)$ & 0.474 \\
\hline 3. Cat Related & 809 & $(10.5)$ & 232 & $(28.7)$ & 9 & $(1.1)$ & 168 & $(20.8)$ & 400 & $(49.4)$ & 0.713 \\
\hline 4. Hit by Car & 483 & $(6.3)$ & 164 & $(34.0)$ & 11 & $(2.3)$ & 65 & $(13.5)$ & 243 & $(50.3)$ & 0.660 \\
\hline
\end{tabular}

${ }^{* *}$ Most common herptiles include chelonia, snakes, amphibians, lizards; most common mammals include rabbits, squirrels, opossums, racoons; most common avian include songbirds, coulumbiformes, raptors, waterfowl

*Trauma includes all other types of trauma (ex: blunt force, entrapment, unknown origin) excluding specifically "Cat Related", "Dog Related", and "Hit by Car"

${ }^{* *} \mathrm{n}$ is the total number of cases in each species group and admitting/presenting signs, (\%) represents the percent of total cases within each species group

Table 2: Case outcomes and case fatality rates (CFR) of the most frequent admitting/presenting signs for herptile,

mammal, and avian species presented to a wildlife clinic in East Tennessee between 2000-2011

Overall mortality was $45.3 \%$ for the herptile cases, $57.6 \%$ for mammal cases, and $65.1 \%$ for avian cases. Many of the A/PSs could be directly attributed to anthropogenic reasons and accounted for 4,328 cases (30.3\%). Reason for case presentation related to habitat disturbance and interactions with humans and domestic pets, in decreasing order, include cat-related, hit by automobile, dog-related, habitat/nest destruction, accidental ingestion (which includes foreign bodies and accidental ingestion of potential poisons), and translocation (Table 1).

Hit by automobile cases had the highest fatality rate (0.7), followed by trauma (0.678), cat-related injury cases (0.675), and dogrelated cases (0.6) for all animal groups. Although cat-related cases had the highest percent of natural deaths following presentation 
to the clinic (18.3\%), hit by automobile cases had the highest percent of cases with successive euthanasia (59.7\%). The animals most frequently represented in cat-related cases were rabbits $(n=757$; mortality $=70.9 \%)$, songbirds $(n=678$; mortality $=69.8 \%)$, and squirrels $(n=225$; mortality $=52.9)$. Animals most frequently represented in hit by automobile cases were chelonia $(n=266$; mortality $=58.7 \%)$, songbirds $(n=176$; mortality $=70.5 \%)$, and rabbits $(n=123$; mortality $=91.1 \%)$. Animals most frequently represented in dog-related cases were rabbits $(n=365$; mortality $=54.3 \%)$, songbirds $(n=186$; mortality $=68.3 \%)$, and squirrels $(n=94$; mortality $=68.1 \%)$.

\begin{tabular}{|l|c|c|c|c|c|c|c|c|c|c|c|}
\hline & \multicolumn{2}{|c|}{$n$} & \multicolumn{2}{|c|}{ ALIVE (\%) } & \multicolumn{2}{c|}{ DOA (\%) } & \multicolumn{2}{|c|}{ DIED (\%) } & \multicolumn{2}{|c|}{ EUTHAN (\%) } & $\begin{array}{c}\text { Mortality } \\
\text { (\%) }\end{array}$ \\
\hline \multicolumn{1}{|c|}{ HERP } & 826 & $(100.00)$ & 452 & $(54.72)$ & 6 & $(0.73)$ & 56 & $(6.78)$ & 312 & $(37.77)$ & 45.28 \\
\hline 1. Chelonia & 743 & $(89.95)$ & 409 & $(55.05)$ & 4 & $(0.54)$ & 50 & $(6.73)$ & 280 & $(37.69)$ & 44.95 \\
\hline 2. Snakes & 57 & $(6.90)$ & 31 & $(54.39)$ & 2 & $(3.51)$ & 5 & $(8.77)$ & 19 & $(33.33)$ & 45.61 \\
\hline 3. Amphibian & 20 & $(2.42)$ & 7 & $(35.00)$ & 0 & $(0.00)$ & 1 & $(5.00)$ & 12 & $(60.00)$ & 65.00 \\
\hline 4. Lizards & 6 & $(0.73)$ & 5 & $(83.33)$ & 0 & $(0.00)$ & 0 & $(0.00)$ & 1 & $(16.67)$ & 16.67 \\
\hline \multicolumn{1}{|c|}{ MAMMAL } & 5761 & $(100.00)$ & 2445 & $(42.44)$ & 65 & $(1.13)$ & 504 & $(8.75)$ & 2747 & $(47.68)$ & 57.56 \\
\hline 1. Rabbit & 2293 & $(39.80)$ & 871 & $(37.99)$ & 25 & $(1.09)$ & 249 & $(10.86)$ & 1148 & $(50.07)$ & 62.01 \\
\hline 2. Squirrel & 1566 & $(27.18)$ & 784 & $(50.06)$ & 11 & $(0.70)$ & 139 & $(8.88)$ & 632 & $(40.36)$ & 49.94 \\
\hline 3. Opossum & 680 & $(11.80)$ & 361 & $(53.09)$ & 6 & $(0.88)$ & 40 & $(5.88)$ & 273 & $(40.15)$ & 46.91 \\
\hline 4. Raccoon & 362 & $(6.28)$ & 117 & $(32.32)$ & 7 & $(1.93)$ & 11 & $(3.04)$ & 227 & $(62.71)$ & 67.68 \\
\hline \multicolumn{1}{|c|}{ AVIAN } & 7716 & $(100.00)$ & 2695 & $(34.93)$ & 88 & $(1.14)$ & 1251 & $(16.21)$ & 3682 & $(47.72)$ & 65.07 \\
\hline 1. Songbird & 4535 & $(58.77)$ & 1559 & $(34.38)$ & 55 & $(1.21)$ & 815 & $(17.97)$ & 2106 & $(46.44)$ & 65.62 \\
\hline 2. Coulumbiformes & 778 & $(10.08)$ & 237 & $(30.46)$ & 9 & $(1.16)$ & 96 & $(12.34)$ & 436 & $(56.04)$ & 69.54 \\
\hline 3. Raptors & 1123 & $(14.55)$ & 469 & $(41.76)$ & 14 & $(1.25)$ & 121 & $(10.77)$ & 519 & $(46.22)$ & 58.24 \\
\hline 4. Waterfowl & 577 & $(7.48)$ & 212 & $(36.74)$ & 3 & $(0.52)$ & 79 & $(13.69)$ & 283 & $(49.05)$ & 63.26 \\
\hline
\end{tabular}

${ }^{* *} \mathrm{n}$ is the total number of cases in each species group and species subgroup, (\%) represents the percent of total cases within each species group

Table 3: Case outcomes and mortality rates (\%) for most common herptile, mammal, and avian species groups presented to a wildlife clinic in East Tennessee between 2000-2011

The cases were relatively evening distributed over the course of the 11 years of data collection. The year and the corresponding contributing percentage to the overall number of cases are as follows: 2000 (11.17\%), 2001 (9.53\%), 2002, (7.64\%), 2003 (8.17\%), 2004 (7.30\%), 2005 (7.40\%), 2006, (7.82\%), 2007 (7.27\%), 2008 (7.66\%), 2009 (9.35\%), 2010 (8.72\%), 2011 (7.97\%) with a standard deviation of 0.01 .

\section{Discussion}

Wildlife species are continually being presented to veterinary clinics throughout the United States, and it is important to study the reasons in order to monitor the changing health status of the surrounding ecosystem [13], decrease the anthropogenic effect of habitat fragmentation and pathogen pollution $[1,3,7,8]$, and investigate preemptive strategies for reducing the number of wildlife casualties. This large dataset provides a good sample to thoroughly explore causal trends for presentation and sheds light on some of the major threats to wildlife health. Although many of the causal factors reported are anthropogenic in origin, the database used in this study also includes physiologic maladies and traumatic injuries unknown in origin. Signs of respiratory, gastrointestinal, neurologic, and other physiologic problems may be the result of some disease process, but evidence of the source and specifics of these diseases or other processes are unclear and, therefore, not investigated. This study does not attempt to explain the origin or cause of all reasons for presentation, but rather focuses on the most frequent causes of presentation.

The most frequent categories-trauma and found/ abandoned/ orphaned do not explicitly imply direct human involvement, but they do highlight the importance of understanding how wildlife habitats are changing in response to both natural and unnatural disruptions. Although pathogen pollution, noise pollution, and environmental pollution, have been shown to lead to wildlife morbidity [1,2,15-17], this study provides an additional explanation that "predator pollution," by means of introducing domestic cats and dogs to wildlife areas, may also be having a profound and damaging effect. This study further supports the importance of reducing the impact human infringement has on native wildlife [18] in order to protect the health of the wildlife and support ecosystems.

With a standard deviation of 0.01 , the percentage of overall cases seen each year did not differ dramatically. This may represent a minimal change in presentation frequency over the course of the eleven years included in this study, but further studies focused on temporal and seasonal trends would be helpful in further studying anthropogenic effects.

The data provided in this study does not investigate or support the role of environmental pollution, pesticide use, or other forms of habitat disruption, but it does lend itself to the needed discussion about the many factors contributing to the morbidity and 
mortality of native wildlife species. In order to establish long-term conservation, a variety of initiatives must be considered. Increasing canopy coverage and the shrub layer along urban parks and greenways has been suggested to increase crucial habitat areas for certain avian species [19]. In addition, evidence supports certain habitat defragmentation projects, such as linear patches and biological corridors, as successful in increasing migratory ranges and establishing connectivity between wildlife populations $[11,20,21]$. On a smaller scale, establishing larger wildlife-friendly areas by arranging neighborhood gardens adjacent to each other has also been proposed as a means to increase wildlife habitat in urbanized areas [22]. By defragmenting habitats and re-establishing connectivity between wildlife populations, the impact of disease spread as well as roadside mortality can possibly be decreased. In addition, if domestic animals and invasive species are removed as predators in the natural order of wildlife ecosystems, the anthropogenic threat to wildlife survival may be significantly reduced.

A need exists for increased community and veterinary client education about the importance, as it relates to wildlife, of keeping domestic cats indoors and preventing domestic dogs from roaming outside unsupervised. Although invasive species may arrive in a given ecosystem from a number of sources, providing educational materials to owners about the proper care of their exotic pets may decrease those introduced to the wild by intentional abandonment. By removing these invasive predators and focusing efforts on the conservation of native habitats, the survivorship of wildlife species can be better protected.

\section{Conclusion}

This study examined the causes for wildlife submission to a wildlife clinic to understand the patterns and trends of reasons for presentation and in order to gain insight to what might be done to mitigate these reasons. Through this and other studies, it is apparent that anthropogenic factors, including land development, habitat fragmentation, as well as direct interactions with automobiles and invasive predators are important causes of wildlife morbidity and mortality. Because final diagnosis was assumed from the A/PS in our study and because some signs lacked explanatory detail, additional studies reviewing comprehensive patient records may provide stronger evidence supporting patterns for wildlife presentation to veterinary clinics. It is also important to understand that many of these cases were submitted by "Good Samaritans" and this may present a bias in the case data. The animals brought to the clinic were likely found in easily accessible, populated areas, and this may lead to an under-representation of the causes of morbidity and mortality in non-developed areas.

Programs to re-establish habitat connectivity such as biological corridors and large neighborhood gardens may serve to protect the biodiversity as well as the survivorship of native species. In addition to defragmenting habitats, it is also important to remove invasive species and contain domestic animals in order to decrease the predatory stress they impose. Through agendas like these and a more mindful approach to land development planning, the anthropogenic threat to wildlife species might be minimized.

\section{References}

1. Daszak P, Cunningham AA, Hyatt AD (2000) Emerging infectious diseases of wildlife-Threats to biodiversity and human health. Science 287: 443-9.

2. Tsipoura N, Burger J, Newhouse M, Jeitner C, Gochfeld M, et al. (2011) Lead, mercury, cadmium, chromium, and arsenic levels in eggs, feathers, and tissues of Canada geese of the New Jersey Meadowlands. Environ Res 111: 775-84.

3. Pavlacky DC Jr, Possingham HP, Lowe AJ, Prentis PJ, Green DJ, et al. (2012) Anthropogenic landscape change promotes asymmetric dispersal and limits regional patch occupancy in a spatially structured bird population. J Ani Ecol 81: 940-52.

4. Robertson ID (1998) Survey of predation by domestic cats. Aust Vet J 76: 551-4.

5. Ament R, Clevenger A, Yu O, Hardy A (2008) An assessment of road impacts on wildlife populations in U.S. national parks. Environ Manage 42: 480-96.

6. Gardner TA, Barlow J, Chazdon R, Ewers RM, Harvey CA, et al. (2009) Prospects for tropical forest biodiversity in a human-modified world. Ecol Lett 12: 561-82.

7. Segelbacher G, Manel S, Tomiuk J (2008) Temporal and spatial analyses disclose consequences of habitat fragmentation on the genetic diversity in capercaillie (Tetrao urogallus). Mol Ecol 17: 2356-67.

8. Leu M, Hanser SE, Knick ST (2008) The human footprint in the west: A large-scale analysis of anthropogenic impacts. Ecol Appl 18: 1119-39.

9. Polley L, Thompson RCA (2009) Parasite zoonoses and climate change: molecular tools for tracking shifting boundaries. Trends Parasitol 25: 285-91.

10. Sehgal RN (2010) Deforestation and avian infectious diseases. J Exp Biol 213: 955-60.

11. Trocmé M (2006) Habitat fragmentation due to linear transportation infrastructure: An overview of mitigation measures in Switzerland. Proceedings of the 6th Swiss Transport Research Conference.

12. Schrader GM, Allender MC, Odoi A (2010) Diagnosis, treatment, and outcome of eastern box turtles (Terrapene carolina carolina) presented to a wildlife clinic in Tennessee, USA, 1995-2007. J Wildl Dis 46: 1079-85.

13. Molina-López RA, Casal J, Darwich L (2011) Causes of morbidity in wild raptor populations admitted at a wildlife rehabilitation centre in Spain from 19952007: a long term retrospective study. PLoS One 6: e24603.

14. Brown JD, Sleeman JM (2002) Morbidity and mortality of reptiles admitted to the Wildlife Center of Virginia, 1991 to 2000. J Wildl Dis 38: 699-705.

15. Maz-Courrau A, López-Vera C, Galván-Magaña F, Escobar-Sánchez O, Rosíles-Martínez R, et al. (2012) Bioaccumulation and biomagnification of total mercury in four exploited shark species in the Baja California Peninsula, Mexico. Bull Environ Contam Toxicol 88: 129-34.

16. Arlettaz R, Patthey P, Baltic M, Leu T, Schaub M, et al. (2007) Spreading free-riding snow sports represent a novel serious threat for wildlife. Proc Biol Sci 274: 1219-24.

17. Romano MC, Rodas AZ, Valdez RA, Hernández SE, Galindo F, et al. (2010) Stress in wildlife species: Noninvasive monitoring of glucocorticoids. Neuroimmunomodulation 17: 209-12.

18. Daszak P, Cunningham AA, Hyatt AD (2001) Anthropogenic environmental change and the emergence of infectious diseases in wildlife. Acta Trop 78: $103-16$. 
19. U.S. Fish and Wildlife Service (2003) Recovery Plan for the Red-cockaded Woodpecker (Picoides borealis): Second Revision, Atlanta, GA, USA.

20. Shepard B, Whittington J (2006) Response of wolves to corridor restoration and human use management. Ecology and Society, USA.

21. Rosenberg DK, Noon BR, Meslow EC (1997) Biological corridors: Form, function, and efficacy. BioScience 47: 677-87.

22. Goddard MA, Dougill AJ, Benton TG (2010) Scaling up from gardens: biodiversity conservation in urban environments. Trends Ecol Evol 25: 90-8.

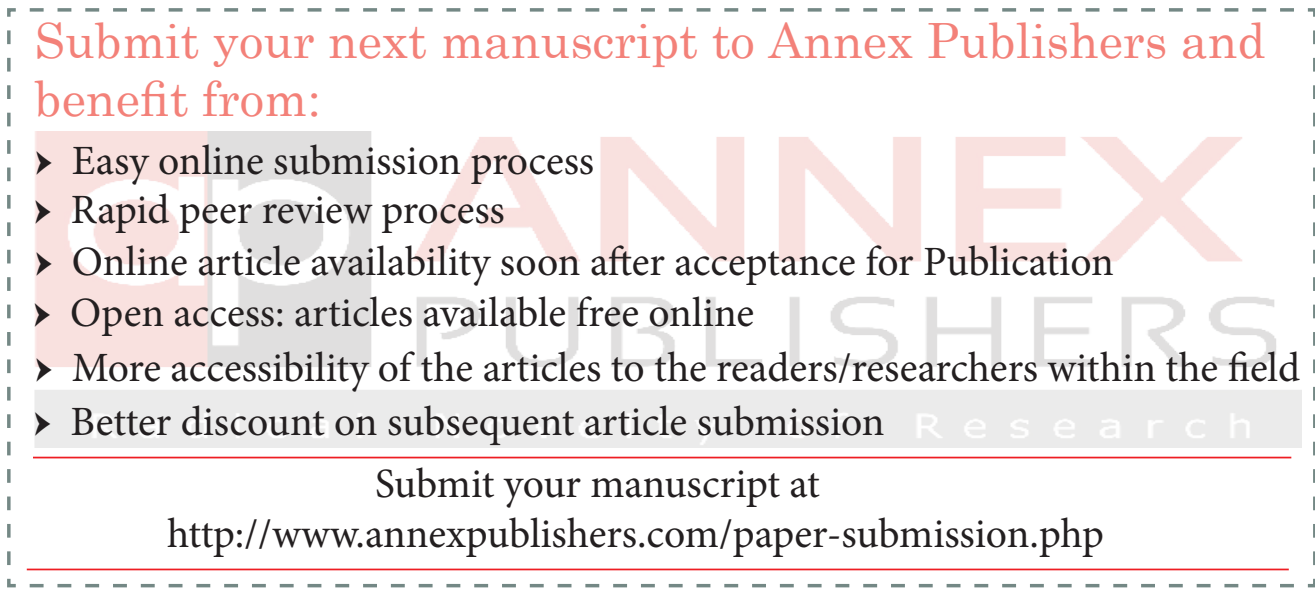

